\title{
Is dopamine transporter invariably impaired at the time of diagnosis in dementia with Lewy bodies?
}

\author{
Flavio Nobili ${ }^{1}$ - Dario Arnaldi ${ }^{1} \cdot$ Silvia Morbelli $^{2}$
}

Received: 17 January 2016 / Accepted: 19 January 2016 / Published online: 9 February 2016

(C) Springer-Verlag Berlin Heidelberg 2016

Assessment of the integrity of the nigrostriatal dopaminergic pathway with ${ }^{123}$ I-ioflupane SPECT has been approved by the Food and Drug Administration and the European Medicines Agency for the differential diagnosis between dementia with Lewy bodies (DLB) and Alzheimer's disease (AD). This has fulfilled a previously unmet need, i.e. to support the clinical diagnosis of DLB that is usually difficult because of overlapping symptoms with $\mathrm{AD}$ and also with frontotemporal lobe degeneration and vascular cognitive impairment. Indeed, by pooling the results from nine clinicopathological studies including 135 DLB patients and 350 non-DLB patients, the average sensitivity of clinical diagnosis was $49 \%$ (range $0-83 \%$ ) and the average specificity (proportion of nonDLB patients correctly diagnosed clinically) was $92 \%$ (range $79-100 \%$ ) [1]. This specificity was considered good but the sensitivity was considered unacceptably low.

The availability of ${ }^{123}$ I-ioflupane SPECT for dopamine transporter (DAT) assessment in the early 2000s prompted a large European multicentre study published in 2007 whose conclusion was that ${ }^{123}$ I-ioflupane SPECT has a good accuracy in detecting dopaminergic impairment both in probable and

This Editorial Commentary refers to the article http://dx.doi.org/10.1007/ s00259-016-3312-x

Flavio Nobili

flaviomariano.nobili@hsanmartino.it

1 Clinical Neurology, Department of Neuroscience (DINOGMI), University of Genoa and IRCCS AOU San Martino-IST, Largo Daneo, 3, 16132 Genoa, Italy

2 Nuclear Medicine Unit, Department of Health Science (DISSAL), University of Genoa and IRCCS AOU San Martino-IST, Genoa, Italy in possible DLB (with clinical diagnosis as the gold standard), achieving about $90 \%$ specificity, similar to clinical diagnosis in previous studies, but a definitely higher sensitivity (77.7\%) thus pointing to this tool as a valuable support for the clinical diagnosis of DLB [2]. The demonstration of nigrostriatal dopaminergic impairment by SPECT or PET was included in the diagnostic criteria published in 2005 merely as a supportive feature [3]. Still a head-to-head comparison between ${ }^{123} \mathrm{I}$ ioflupane SPECT and neuropathology was needed. This was performed by Walker et al. [4] who found a specificity of $100 \%$ and a sensitivity of $88 \%$ with semiquantitative analysis of the posterior putamen. In early 2015 a Cochrane review of DAT SPECT concluded that DAT SPECT is more accurate than clinical diagnosis in DLB and that clinical diagnosis is unsuitable as a reference standard for assessing the accuracy of DAT imaging [5].

However, the $88 \%$ sensitivity found by Walker et al. suggests that one in eight DLB patients with confirmed DLB pathology at autopsy might have a normal ${ }^{123}$ I-ioflupane SPECT scan. The patient with a normal scan actually had an asymmetric presentation with right-sided parkinsonism resembling a corticobasal syndrome, with abnormal posterior left putamen uptake on visual analysis but normal average counts between the two putamina (the index considered in the paper). On autopsy only mild neuronal loss with mild $\alpha$ synuclein pathology were found in the substantia nigra (SN) of that patient, together with widespread tau tangle/neuritic pathology in both the putamen and caudate. Also, the patient was affected by diffuse DLB pathology in the neocortex, and the likelihood of clinical DLB was retrospectively estimate to be 'intermediate' according to the DLB criteria. After publication of that study, the opinion in the scientific community was that ${ }^{123}$ I-ioflupane SPECT was virtually always positive in all patients with true DLB and that the limited sensitivity shown in the 2007 multicentre study $(77.7 \%$ ) was mainly due 
to the imperfect gold standard, i.e. clinical diagnosis, that has limited accuracy.

In 2011 a neuropathological study conducted in patients with DLB, AD and Parkinson's disease dementia (PDD) showed that ${ }^{123}$ I-ioflupane SPECT correlated fairly well with neuronal density in the SN but not with the amount of $\alpha$-synuclein, tau or amyloid pathology in the SN on post-mortem examination [6], although the authors commented that the lack of correlation with the amount of nigral $\alpha$-synuclein and tau pathology might have been due to the relatively low number of patients. In that neuropathological series 2 of the 19 patients with DLB or PDD actually had a normal scan and a normal or slightly reduced SN neuronal density. The two patients belonged to the DLB group that included seven patients. The authors commented that those individuals who exhibited a greater level of nigral neurons than the other patients in the Lewy body cohort (i.e. the 19 patients with either DLB or PDD) expressed cortical and striatal Lewy body pathology but without significant nigrostriatal neuronal loss, thus still had a 'false-negative' ${ }^{123}$ I-ioflupane SPECT scan. Similar results were also later found by an independent research group that found that 3 of 20 patients who fulfilled the clinical criteria for probable DLB had a normal ${ }^{123}$ I-ioflupane SPECT scan, as evaluated by visual analysis only, while the other 17 patients with probable DLB had a positive scan. The diagnosis of probable DLB was confirmed on clinical follow-up, but the patients did not undergo a repeat ${ }^{123}$ I-ioflupane SPECT scan nor was pathological confirmation performed. Patients with a negative scan did not differ from those with a positive scan in terms of the presence of parkinsonism, but they did not show neuroleptic sensitivity [7]. Of note, in the same study seven patients who were not attributed to the DLB cluster actually had a positive ${ }^{123}$ I-ioflupane scan and all of them developed probable DLB during the clinical follow-up, thus demonstrating the ability of dopaminergic SPECT to pick up nigrostriatal degeneration early before the clinical picture has become clear in a substantial proportion of DLB patients.

Therefore, it appears that a small but not insignificant proportion of DLB patients (possibly about the $10 \%$ ) can have a normal ${ }^{123}$ I-ioflupane SPECT scan when they are diagnosed for the first time. What was not clear until the publication of the article by van der Zande et al. in this issue of the European Journal of Nuclear Medicine and Molecular Imaging [8] is whether their scans remain negative during follow-up or whether they become positive over time. In their remarkable study, van der Zande et al. found that 7 of 67 patients with probable DLB (about $10 \%$ ) had a negative ${ }^{123}$ I-ioflupane SPECT scan as evaluated by semiquantitative analysis. Among these seven patients parkinsonism was significantly less frequent; the diagnosis was confirmed during an average clinical follow-up of 2 years (interquantile range $8-36$ months). The authors were able to perform a repeat scan in five of the seven patients after a median time of 18 months (range $9-38$ months) and the scans were abnormal in all patients, thus proving that SN neuronal density can still be normal at the time of diagnosis, especially in patients without or with mild parkinsonism, but then the spreading of pathology invariably leads to $\mathrm{SN}$ involvement and a positive ${ }^{123} \mathrm{I}-$ ioflupane SPECT scan.

This notion is of paramount clinical relevance as up to now the fate of patients with clinically probable DLB but a negative ${ }^{123}$ I-ioflupane scan has been unclear. In clinical practice this means that a negative scan should not deny the diagnosis of DLB if the patient meets the criteria for probable DLB. Should we then wait a couple of years before repeating the scan to confirm the diagnosis? This seems now the key question. Do we have answers? First, the clinical diagnosis of probable DLB has a poor sensitivity (about $50 \%$ ) but a good specificity (about $90 \%$; that is, if a dementia or movement disorder expert considers an individual patient to be affected with probable DLB the likelihood he/she is right on the basis of neuropathological studies is about $90 \%$ ). If the patient had a normal ${ }^{123} \mathrm{I}$ ioflupane SPECT scan we still might be confident that the patient actually has DLB. This may be confirmed by other diagnostic tools without waiting for two more years before repeating the ${ }^{123}$ I-ioflupane SPECT scan. Indeed, ${ }^{123} \mathrm{I}$ metaiodobenzylguanidine (MIBG) cardiac planar scintigraphy has shown high sensitivity in detecting norepinephrine transporter deficit at the cardiac level even in DLB patients without autonomic failure [9], and the likelihood that a patient with probable DLB has both a normal ${ }^{123}$ I-ioflupane SPECT scan and a normal ${ }^{123}$ I-MIBG scan should be exceedingly rare. Alternative tools are ${ }^{18} \mathrm{~F}$-FDG PET that often shows much more pronounced hypometabolism in the occipital cortex than in $\mathrm{AD}$ patients and patients with other dementias [10]. Moreover, as compared to AD, FD -PET shows relatively preserved uptake in the precuneus/posterior cingulate region in DLB, the so-called 'cingulate island' sign [10]. Finally, quantitative electroencephalography (qEEG), a simple, noninvasive and cheap tool can show significantly higher variability of posterior dominance frequency in DLB patients than in AD patients [11]. Figure 1 shows the expected biomarker features in a representative DLB patient and an AD patient for comparison.

In this context, a preserved nigrostriatal dopaminergic SPECT or PET scan is regarded as an accurate biomarker to exclude a diagnosis of DLB in patients with moderately severe dementia and a strong clinical suspicion of DLB (i.e., probable DLB) [5]. However, the accuracy of these biomarkers in the earliest stages as well as their temporal cascade still needs to be clarified. This issue has been exploited in AD but now is also emerging in DLB, given the concerted effort to establish the phenotypes of the main neurodegenerative dementia in the prodromal phase [12]. This will provide a critical opportunity for potential intervention with disease-modifying drugs [13].

Given the crucial role of ${ }^{123}$ I-ioflupane SPECT findings in early DLB, an issue that needs to be emphasized and is 

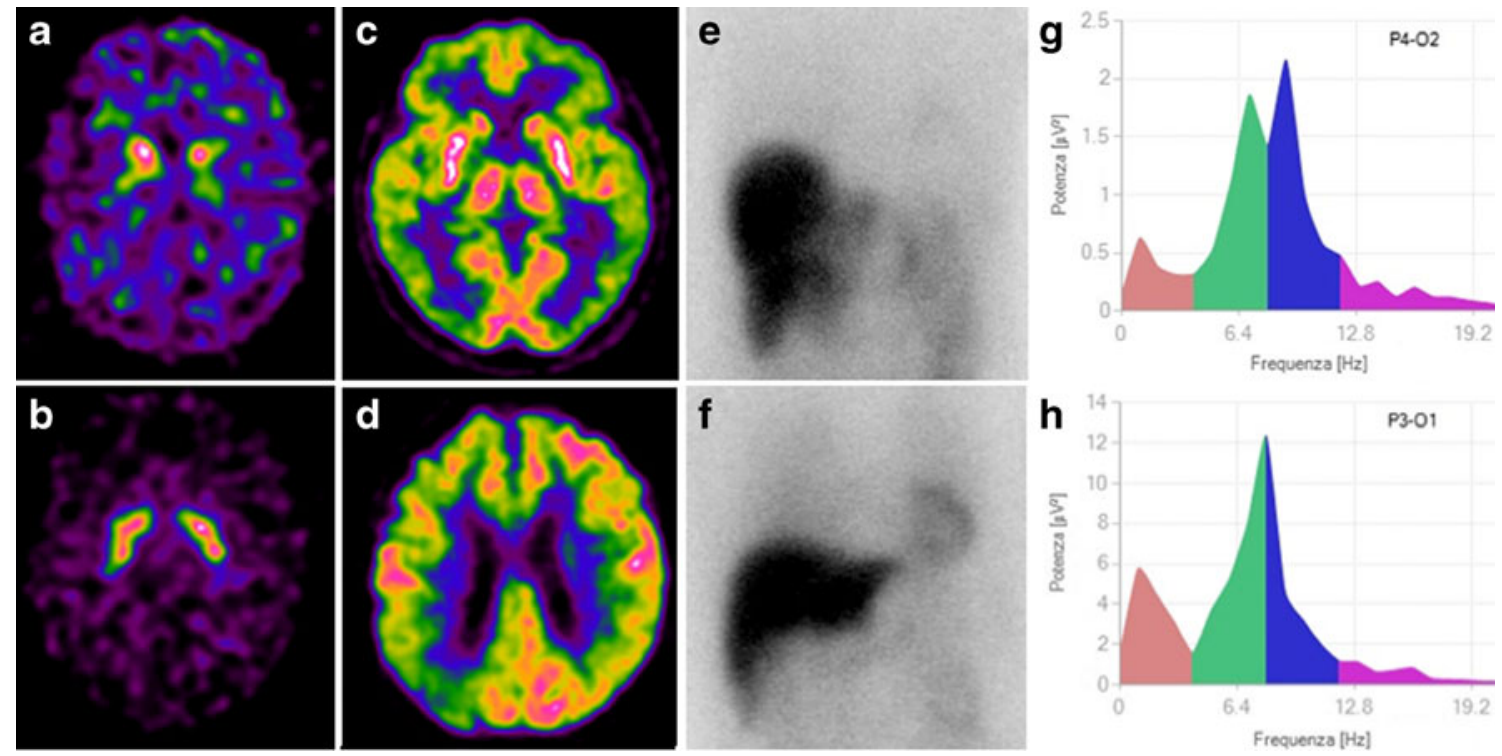

Fig. 1 Biomarker features in a representative patient with mild DLB (MMSE score 23; top row) as compared with those in a similar patient with mild AD (bottom row). a, b ${ }^{123}$ I-ioflupane SPECT images show markedly impaired DAT uptake in the DLB patient (a) but preserved uptake in the AD patient (b). c, d ${ }^{18}$ F-FDG PET images show impaired uptake in the posterior associative cortex as well as in the ventrolateral frontal cortex in the DLB patient (c), with the main involvement of the lateral occipital cortex; in the AD patient (d) hypometabolism in the right superior parietal lobule, posterior cingulate and precuneus can easily be appreciated. e, $\mathbf{f}^{123}$ I-MIBG cardiac planar scintigraphy images cannot identify the heart shadow in the DLB patient (e) which is readily evident in the AD patient (f). $\mathbf{g}, \mathbf{h}$ qEEG power spectrum analysis of the main EEG frequencies highlights two frequency peaks in the posterior dominant frequency (PDF) in the DLB patient (g), peaking at about 7 and $9 \mathrm{~Hz}$, meaning fluctuation of the PDF in the course of the same recording lasting $20 \mathrm{~min}$, while in the AD patient (h) the PDF is at the lower frequency limit (peaking at about $8.5 \mathrm{~Hz}$ ) but without substantial fluctuation especially relevant in nuclear medicine is how the scan is read and reported. While visual assessment by an expert is the basis of all reports, semiquantitative assessment (better if performed with the aid of automatic tools and compared with an adequate normal database) may further increase diagnostic confidence. To this purpose, the Neuroimaging Committee of the EANM in recent years has generated a large normal database of ${ }^{123} \mathrm{I}-$ ioflupane SPECT scans assessed by some of the most popular automatic tools which may be of help in routine clinical practice $[14,15]$.

To summarize, the paper by van der Zande et al. in this issue of the EJNMMI [8] confirms the high sensitivity (about $90 \%$ versus clinical diagnosis) of ${ }^{123}$ I-ioflupane SPECT in demonstrating nigrostriatal dopaminergic impairment in patients with probable DLB. The small proportion of patients (about $10 \%$ ) with a normal scan but still with a strong suspicion of probable DLB on clinical grounds may not have sufficient nigral neuronal loss to have a positive scan while having diffuse cortical and striatal $\alpha$-synuclein pathology, thus with an unusual top-bottom propagation of pathology instead of the reverse, as is usual. In the present state of knowledge, these patients should invariably show a positive scan about $1.5-3$ years later, thus raising the final sensitivity of ${ }^{123} \mathrm{I}-$ ioflupane SPECT to $100 \%$. These patients are less likely to be affected by parkinsonism and to show neuroleptic sensitivity. DLB diagnosis could be confirmed using other molecular imaging tools, such as ${ }^{123}$ I-MIBG cardiac scintigraphy and brain FDG PET, but other tools, such as qEEG, deserve to be considered.

\section{References}

1. Litvan I, Bhatia KP, Burn DJ, Goetz CG, Lang AE, McKeith I, et al. Movement Disorders Society Scientific Issues Committee report: SIC Task Force appraisal of clinical diagnostic criteria for Parkinsonian disorders. Mov Disord. 2003;18:467-86.

2. McKeith I, O'Brien J, Walker Z, Tatsch K, Booij J, Darcourt J, et al. Sensitivity and specificity of dopamine transporter imaging with 123I-FP-CIT SPECT in dementia with Lewy bodies: a phase III, multicentre study. Lancet Neurol. 2007;6:305-13.

3. McKeith IG, Dickson DW, Lowe J, Emre M, O'Brien JT, Feldman $\mathrm{H}$, et al. Diagnosis and management of dementia with Lewy bodies: third report of the DLB Consortium. Neurology. 2005;65:1863-72.

4. Walker Z, Jaros E, Walker RW, Lee L, Costa DC, Livingston G, et al. Dementia with Lewy bodies: a comparison of clinical diagnosis, FP-CIT single photon emission computed tomography imaging and autopsy. J Neurol Neurosurg Psychiatry. 2007;78:1176-81.

5. McCleery J, Morgan S, Bradley KM, Noel-Storr AH, Ansorge O, Hyde C. Dopamine transporter imaging for the diagnosis of dementia with Lewy bodies Dopamine transporter imaging for the diagnosis of dementia with Lewy bodies. Cochrane Database Syst Rev. 2015;1, CD010633.

6. Colloby SJ, McParland S, O'Brien JT, Attems J. Neuropathological correlates of dopaminergic imaging in Alzheimer's disease and Lewy body dementias. Brain. 2012;135:2798-808.

7. Siepel FJ, Rongve A, Buter TC, Beyer MK, Ballard CG, Booij J, et al. (123I)FP-CIT SPECT in suspected dementia with Lewy 
bodies: a longitudinal case study. BMJ Open. 2013;3:e002642. doi: 10.1136/bmjopen-2013-002642.

8. van der Zande J, Booij J, Scheltens P, Raijmakers P, Lemstra AW. [123]FP-CIT SPECT scans initially rated as normal became abnormal over time in patients with probable dementia with Lewy bodies. Eur J Nucl Med Mol Imaging. 2016. doi:10.1007/s00259-0163312-x

9. Chung EJ, Kim SJ. (123)I-metaiodobenzylguanidine myocardial scintigraphy in Lewy body-related disorders: a literature review. J Mov Disord. 2015;8:55-66.

10. Graff-Radford J, Murray ME, Lowe VJ, Boeve BF, Ferman TJ, Przybelski SA, et al. Dementia with Lewy bodies: basis of cingulate island sign. Neurology. 2014;83:801-9.

11. Bonanni L, Thomas A, Tiraboschi P, Perfetti B, Varanese S, Onofrj M. EEG comparisons in early Alzheimer's disease, dementia with Lewy bodies and Parkinson's disease with dementia patients with a 2-year follow-up. Brain. 2008;131(Pt 3):690-705.
12. Donaghy PC, McKeith IG. The clinical characteristics of dementia with Lewy bodies and a consideration of prodromal diagnosis. Alzheimers Res Ther. 2014;6:46.

13. Fujishiro H, Nakamura S, Sato K, Iseki E. Prodromal dementia with Lewy bodies. Geriatr Gerontol Int. 2015;15:817-26.

14. Varrone A, Dickson JC, Tossici-Bolt L, Sera T, Asenbaum S, Booij $\mathrm{J}$, et al. European multicentre database of healthy controls for [123I]FP-CIT SPECT (ENC-DAT): age-related effects, gender differences and evaluation of different methods of analysis. Eur J Nucl Med Mol Imaging. 2013;40:213-27.

15. Nobili F, Naseri M, De Carli F, Asenbaum S, Booij J, Darcourt J, et al. Automatic semi-quantification of [123I]FP-CIT SPECT scans in healthy volunteers using BasGan version 2: results from the ENC-DAT database. Eur J Nucl Med Mol Imaging. 2013;40: $565-73$. 